tures with papillary pattern were noted along the wall of the cyst, and diagnosed papillary adenoma of thyroid origin. The colloidal fluid in the cyst contained $110 \gamma / \mathrm{dl}$ of PBI, of which $9.6 \%$ was organic. The tumor was biochemically an abnormal thyroid tissue. Nothing abnormal was noticed in her cervical region. $\mathbf{I}^{131}$-scintigram appeared normal, and no abnormal uptake of $\mathbf{I}^{131}$ was found in the head and chest. She discharged a month after operation and followed up. Eight months after craniotomy, repeated ${ }^{131}$-scintigram of the thyroid revealed a cold area in the left lobe. She again admitted and operation was performed. A small nodule, measuring $4 \mathrm{~mm}$ in diameter, was discovered in the left lobe of the thyroid with minute metastasis in an adjacent lymph node. Left radical lobectomy was performed. Histologically, the nodule in the thyroid was papillary adenocarcinoma, less differentiated than the metastatic frontal lobe tumor, and considered as the primary lesion.

\title{
13. A Postoperative Long Survival Case of Intracranial Sarcoma. Report of a Case
}

\author{
Naoki Kageyama and Kimiyuki IKedA \\ Dept. of Neurosurgery, Kansai Medical College \\ Hisashi OYA \\ Pediatric Dept., Kitano Hospital
}

Most of intracranial sasrcomas recur within 1-2 years after surgical removal and the prognosis is almost always unfavorable. A case of an intracranial sarcoma who has survived for 6 and a half years postoperatively is reported. The clinical history is as follows:

Case report: The patient was an 11-year-old girl who was admitted to the 1st Surgical Division of Kyoto University Hospital on March 14, 1961 because of frequent spells of awkwardness and weakness of the right half of the body accompanied by paresthetic feeling in the right lower extremity for about 5 month duration. Since four months ago she has complained nausea and headache.

Neurological examination revealed tenderness on percussion in the left parietal area, right spastic hemiparesis and positive bilateral Babinski sign. Skull plain films showed a calcified mass of walnut-size in the left parietal area. Left carotid angiogram revealed a space occupying lesion in the corresponding area of the calcification. Left parietal craniotomy was done on March 18, 1961. A sharply demarcated calcified tumor was found in the parietal parasagittal area and partly attached to the dura. The tumor was carefully separated from the surrounding brain tissue and extirpated in toto en block. After the operation the patient has 
been completely well and in good health for 6 and a half years.

The tumor, weighed $48.5 \mathrm{~g}$, is of dumb-bell form. One part is hard and another soft. Microscopically the hard part presents a striking contrast to the soft part. The former is mainly composed of mature bone tissue accompanied by canalicular formation and interstitial connective tissue stroma. The latter is, however, a very cellular tumor which is mainly composed of spindle shaped cells with a hyperchromatic nucleus and scanty cytoplasm. Pleomorphism is not so marked, but mitotic figures are numerous. A reticulum fiber stain section shows numerous fine strands of reticulum throughout the tumor. This part is diagnosed as a fibrosarcoma.

From the above histological findings, it is considered that the fibrosarcoma originated from the preexisting teratoma or osteoma.

\title{
14. A Case of Sarcoma following Postoperative Radiation Therapy for Ependymoma
}

\author{
Tohru Higashi, Hikozo Shimada, Minoru Arimori \\ and Norihiko MizuKawa \\ Department of Neurological Surgery, Okayama University Medical School
}

There are a number of reported cases of sarcoma in the path of x-ray beams following postoperative radiation treatment.

We have experienced a case in which a fibrosarcoma developed 4 years after radiation therapy in the region where a brain tumor was removed totally.

This 9 year-old boy was admitted on May 25, 1963 with a diagnosis of brain tumor because of headache and vomiting followed by the left hemiplegia of threemonth duration. At operation on June 5, an ependymoma with a cyst was found in the right frontal lobe and removed. In postoperative radiotherapy a tumor dose of 3,000 $\mathrm{R}$ was given. The patient remained entirely well until August, 1966, when the symptoms recurred. A second radiotherapy was given with a good effect. The patient was again seen in April, 1967 with symptoms of raised intracranial pressure and underwent a second operation. A fibrosarcoma, $150 \mathrm{~g}$ in weight, was found in the region in which the previous operation of a total removal of the tumor was performed. At operation the tumor was found to be isolated from the dura. The postoperative course was uneventful except for weakness in the left upper extremity until August, 1967, when a third operation was performed because of recurrence of symptoms. At operation fibrosarcoma was found to occupy the right parietal and frontal lobes and a total removal of $300 \mathrm{~g}$ tumor was performed. At the present time, he can walk and lives well with mild left hemiplegia. 